\title{
Magnetism in (III, Mn)-V Diluted Magnetic Semiconductors: Effective Heisenberg Model
}

\author{
S. Hilbert ${ }^{1}$, , and W. Nolting ${ }^{2}$ \\ ${ }^{1}$ Max-Planck-Institut für Astrophysik, Karl-Schwarzschild-Straße 1, D-85741, Garching, Germany \\ ${ }^{2}$ Institut für Physik, Humboldt-Universität zu Berlin, Newtonstraße 15, D-12489 Berlin, Germany
}

(Dated: November 8, 2018)

\begin{abstract}
The magnetic properties of the diluted magnetic semiconductors (DMS) (Ga, Mn)As and (Ga, $\mathrm{Mn}) \mathrm{N}$ are investigated by means of an effective Heisenberg model, whose exchange parameters are obtained from first-principle calculations. The finite-temperature properties of the model are studied numerically using a method based upon the Tyablikov approximation. The method properly incorporates the effects of positional disorder present in DMS. The resulting Curie temperatures for $(\mathrm{Ga}, \mathrm{Mn}) \mathrm{As}$ are in excellent agreement with experimental data. Due to percolation effects and noncollinear magnetic structures at higher Mn concentrations, our calculations predict for (Ga, $\mathrm{Mn}) \mathrm{N}$ very low Curie temperatures compared to mean-field estimates.
\end{abstract}

PACS numbers: 75.50.Pp, 75.10.Nr, 85.75.-d

\section{INTRODUCTION}

Ferromagnetic (III, Mn)-V diluted magnetic semiconductors (DMS) have attracted considerable attention among scientist during the past years 1.2 . Their investigation has been driven by the idea of using their coupled electronic and magnetic degrees of freedom to construct electronic devices ranging from fast nonvolatile memories to quantum computers ${ }^{3}$. To date, however, technical applicability has been limited by the fact that most known DMS have Curie temperatures $T_{C}$ below room temperature ${ }^{2,4,5,6,7}$.

For the development of ferromagnetic DMS with higher Curie temperatures, it is important to understand theoretically the magnetism in these materials and to develop theories which provide reliable qualitative and quantitative predictions. The magnetism in these materials is due to magnetic moments localized at magnetic impurities, which interact with each other indirectly via holes in the valence and impurity band of the host semiconductor. Therefore, for the description, one often employs an effective Heisenberg model, whose exchange parameters are determined by the interaction between the localized moments and the holes 8.9 .10 .11 .12 .13 .14 .15$. However, the magnetic impurities are mainly randomly distributed over the sites of the crystal lattice. This positional disorder breaks the translational symmetry of the crystal and thus greatly complicates the theoretical description of the material. Studies based on the mean-field approximation (MFA) ${ }^{8.9}$ or the random-phase approximation (RPA) combined with the virtual-crystal approximation $(\mathrm{VCA})^{10}$ neglect effects of the positional disorder in DMS. Approaches based on percolation theory ${ }^{11.12}$ account for the randomness of the impurity positions, but require a simple functional dependence of the exchange parameters on the inter-spin distance and treat the magnetism itself only on a mean-field level. Monte-Carlo simulations $(\mathrm{MC})^{13.14 .15 .16}$ seem to provide a better way to include the positional disorder, but these are numerically expensive and usually assume classical spins. However, a proper treatment of the positional disorder of the localized moments and their quantum nature is needed to make reliable predictions about the magnetic properties of $\mathrm{DMS}^{17.18}$.

In a previously published article ${ }^{9}$, the exchange parameters of an effective (classical) Heisenberg Hamiltonian have been calculated from first-principles for $\mathrm{Ga}_{1-x} \mathrm{Mn}_{x}$ As and $\mathrm{Ga}_{1-x} \mathrm{Mn}_{x} \mathrm{~N}$. There, however, these had only been used to calculate Curie temperatures within MFA. More recently, results of classical MC simulations on the basis of these exchange parameters have been presented ${ }^{15}$. Here, we employ a different approach ${ }^{19,20}$ to investigate the properties of the effective Heisenberg Hamiltonian. This approach generalizes the Tyablikov approximation ${ }^{21}$ to systems with positional disorder, which is treated numerically exactly. Furthermore, the method assumes quantum spins. The quantum fluctuations of the spins are treated within randomphase approximation, which goes beyond MFA and the classical-spin approximation. It should be mentioned that a similar approach has been proposed in ${ }^{22}$.

\section{MODEL}

Details of the electronic-structure calculation for $\mathrm{Ga}_{1-x} \mathrm{Mn}_{x}$ As and $\mathrm{Ga}_{1-x} \mathrm{Mn}_{x} \mathrm{~N}$ and the extraction of the exchange parameters $J(\mathbf{R})$ as function of the Mn-Mn distance $\mathbf{R}$ can be found in Ref $\stackrel{9}{~}$. Here, these exchange parameters are used as input for a 'diluted' Heisenberg model

$$
H=-\sum_{i, j=1}^{N} J_{i j} \mathbf{e}_{i} \cdot \mathbf{e}_{j},
$$

in which only a fraction of the lattice sites is occupied by a spin. Hence, $i$ and $j$ label the occupied lattice sites only, whose total number is $N$, and $\mathbf{e}_{i}=\left(S_{i}^{x}, S_{i}^{y}, S_{i}^{z}\right) /(\hbar S)$ 
is the normalized spin operator of the localized magnetic moment at lattice site $i$ with lattice vector $\mathbf{R}_{i}$ and $J_{i j}=J\left(\left|\mathbf{R}_{\mathbf{i}}-\mathbf{R}_{j}\right|\right)$. The magnitude $S$ of the spins is absorbed by the exchange parameters due to the particular way in which these are calculated from the electronic structure.

The finite-temperature properties of Hamiltonian (11) are studied using a generalization of the Tyablikov approximation to systems without translational symmetry ${ }^{19,20}$. The generalization treats the positional disorder in the spin system numerically exactly except that a uniform magnetization is assumed. Furthermore, the effects of low-energy quantum excitations, i.e. magnons, are included. Within this approximation, the local magnon spectral density is given by ${ }^{19,20}$ :

$$
S_{i i}(E)=2 \hbar^{2}\left\langle S^{z}\right\rangle \frac{1}{N} \sum_{r=1}^{N} \delta\left(E-\frac{2 \hbar\left\langle S^{z}\right\rangle}{\hbar^{2} S^{2}} E_{r}\right),
$$

where the $E_{r}$ are the eigenvalues of the Hamilton matrix $\mathbf{H}$, which is defined by its matrix elements $H_{i j}=\delta_{i j} \sum_{n=1}^{N} J_{i n}-J_{i j}$. These eigenvalues also determine the Curie temperature:

$$
k_{B} T_{C}=\frac{2}{3} \frac{S(S+1)}{S^{2}}\left(\frac{1}{N} \sum_{r} \frac{1}{E_{r}}\right)^{-1} .
$$

To evaluate this expression for a given set of $E_{r}$ 's, the value of $S$ has to be fixed. For Mn ions in $\mathrm{Ga}_{1-x} \mathrm{Mn}_{x} \mathrm{As}$ and $\mathrm{Ga}_{1-x} \mathrm{Mn}_{x} \mathrm{~N}, S=5 / 2$ should be appropriate ${ }^{2}$. However, this choice is not consistent with the calculation of the exchange parameters from the electronic structure, where classical spins are assumed. Therefore, we will use Eq. (3) in the limit $S \rightarrow \infty$, which yields $T_{C}$ values a factor $5 / 7$ less than for $S=5 / 2$.

Due to the positional disorder of the spins present in DMS, the eigenvalues cannot be computed by Fourier transformation of $\mathbf{H}$. However, the eigenvalues may be obtained by the numerical diagonalization of the Hamilton matrix for a finite system. In our calculations, we used systems of $\sim 10000$ spins, which were randomly distributed over the lattice sites of a cubic section of an fcc lattice with periodic boundary conditions. For each concentration $x$ of Mn ions, we averaged the spectral densities over eight random configurations.

\section{RESULTS}

In Fig. 1 the Mn-Mn exchange interactions $J(\mathbf{R})$ in $\mathrm{Ga}_{1-x} \mathrm{Mn}_{x} \mathrm{As}$ and in $\mathrm{Ga}_{1-x} \mathrm{Mn}_{x} \mathrm{~N}$ are shown as functions of the Mn-Mn distance $R$ for several concentration $x$. In $\mathrm{Ga}_{1-x} \mathrm{Mn}_{x} \mathrm{As}$, the falloff of the interaction with $R$ is comparably slow. In $\mathrm{Ga}_{1-x} \mathrm{Mn}_{x} \mathrm{~N}$, the interaction between nearest neighbors is much larger than in $\mathrm{Ga}_{1-x} \mathrm{Mn}_{x} \mathrm{As}$, but Mn moments further apart are only very weakly coupled.
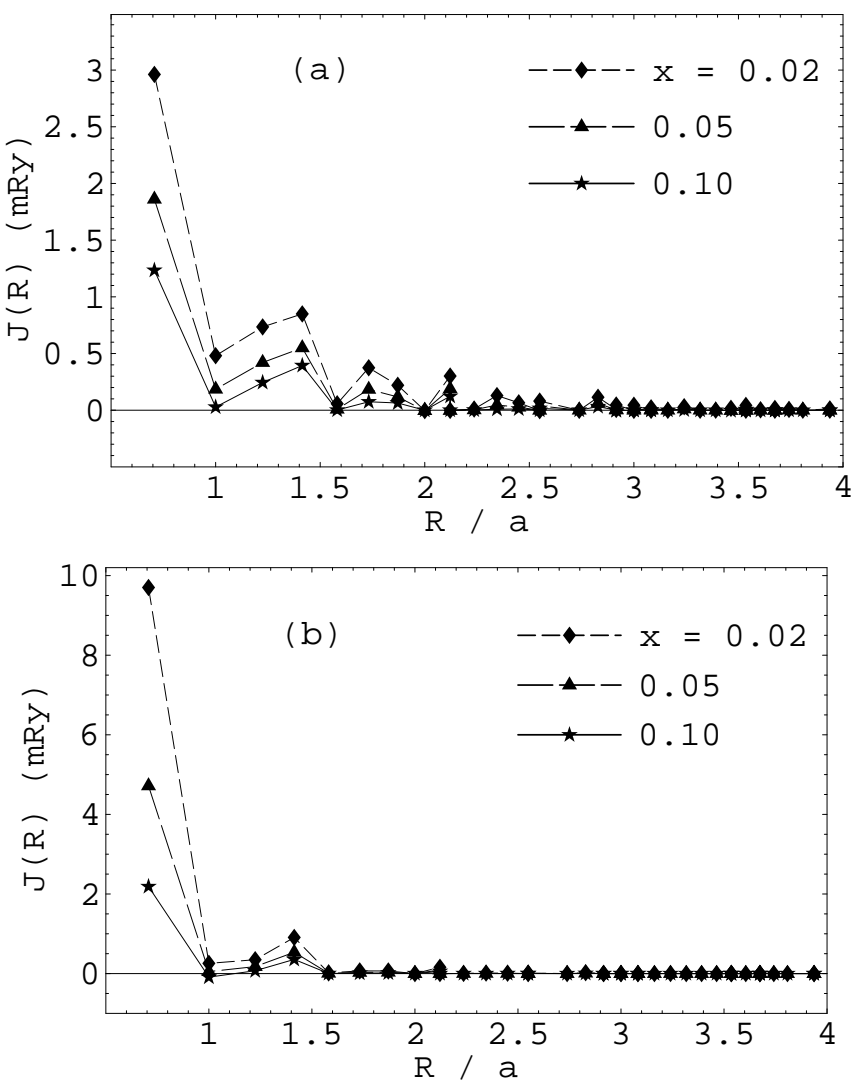

FIG. 1: Exchange interactions $J(\mathbf{R})$ between Mn ions of distance $\mathbf{R}$ in (a) $\mathrm{Ga}_{1-x} \mathrm{Mn}_{x} \mathrm{As}$ and (b) $\mathrm{Ga}_{1-x} \mathrm{Mn}_{x} \mathrm{~N}$ for various concentrations $x$ (from ${ }^{9.23}$ )

Figure 2 shows the resulting magnon spectral densities. For $\mathrm{Ga}_{1-x} \mathrm{Mn}_{x} \mathrm{As}$, the spectrum is smooth and continuous. For $\mathrm{Ga}_{1-x} \mathrm{Mn}_{x} \mathrm{~N}$, one can recognize remnants of peaks typical for nearest-neighbor interaction at low concentrations, which are broadened by small longranged interactions. Compared to $\mathrm{Ga}_{1-x} \mathrm{Mn}_{x} \mathrm{As}$, there is a large spectral density at low energies for $\mathrm{Ga}_{1-x} \mathrm{Mn}_{x} \mathrm{~N}$. For concentrations $x \geq 0.08$, antiferromagnetic interactions come into play and negative magnon energies appear indicating a ground state which is not a saturated ferromagnet ${ }^{20}$.

The Curie temperatures calculated using Eq. (3) are shown in Fig. 3 For $\mathrm{Ga}_{1-x} \mathrm{Mn}_{x} \mathrm{As}$, the calculated values agree remarkably well with the experimental values of optimally annealed samples,6.24. Furthermore, the calculated curve suggests that slightly higher $T_{C}$ 's might be achieved by further increasing the Mn content $x$, but values above $300 \mathrm{~K}$ seem rather unlikely.

Since experimental values for $T_{C}$ in $\mathrm{Ga}_{1-x} \mathrm{Mn}_{x} \mathrm{~N}$ are quite controversial (reported values range from $0 \mathrm{~K}$ to $940 \mathrm{~K}^{4,26,27,28,29,30}$ ), we refrain from a comparison here. However, the Curie temperatures we calculated are quite low compared to earlier mean-field estimates (e.g. in Ref ${ }^{2}$ ). These low $T_{C}$ values despite the high values of the nearest-neighbor exchange may be explained as fol- 

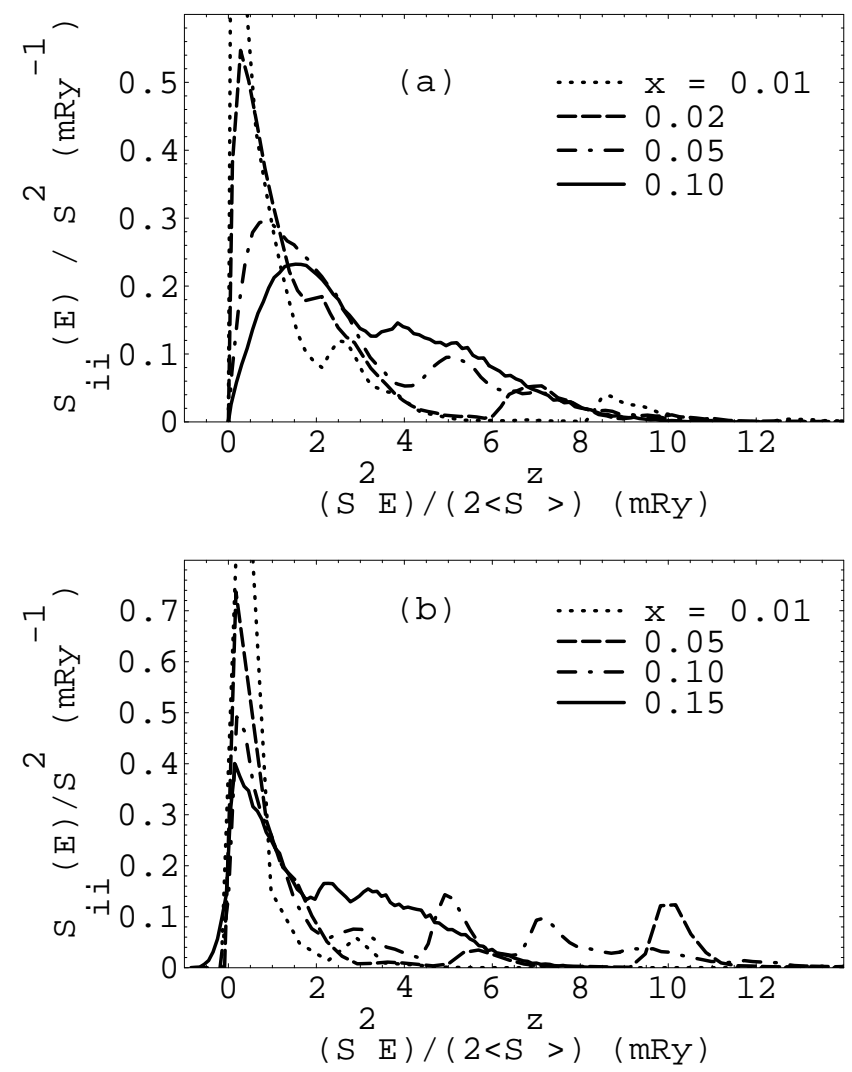

FIG. 2: Local magnon spectral density $S_{i i}(E)$ for (a) $\mathrm{Ga}_{1-x} \mathrm{Mn}_{x}$ As and (b) $\mathrm{Ga}_{1-x} \mathrm{Mn}_{x} \mathrm{~N}$ for various concentrations $x$ of $\mathrm{Mn}$

lows: For concentrations well below the nearest-neighbor percolation threshold $c_{P} \approx 0.2^{31}$, even a large nearestneighbor exchange does not contribute substantially to the stability of the magnetic phase. Since the exchange parameters for larger inter-spin distances are very small in $\mathrm{Ga}_{1-x} \mathrm{Mn}_{x} \mathrm{~N}$, ferromagnetic order can only be established at very low temperatures. Note that the drop of $T_{C}$ for $x \geq 0.08$ may be due to the used approximation: As indicated by the magnon spectra seen in Fig. 2 the system's ground state is different from a saturated ferromagnet, but a such uniform magnetic state is assumed in the approximation.

Figure 4 presents a comparison of the the Curie temperatures calculated using different approximations for the effective Heisenberg model. The $T_{C}$ values obtained by $\mathrm{MC}$ simulations are slightly higher than the ones calculated by the presented approach, whereas both MFA and VCA-RPA yield much higher $T_{C}$ 's. For $\mathrm{Ga}_{1-x} \mathrm{Mn}_{x} \mathrm{As}$, the difference is about a factor two to eight. For $\mathrm{Ga}_{1-x} \mathrm{Mn}_{x} \mathrm{~N}$, the difference is even much larger. This is due to the fact that the MFA and VCARPA do not take into account percolation effects: Large nearest-neighbor interactions yield large Curie temperatures even for concentrations well below the nearestneighbor percolation threshold. However, for such concentrations, the nearest-neighbor interaction strength
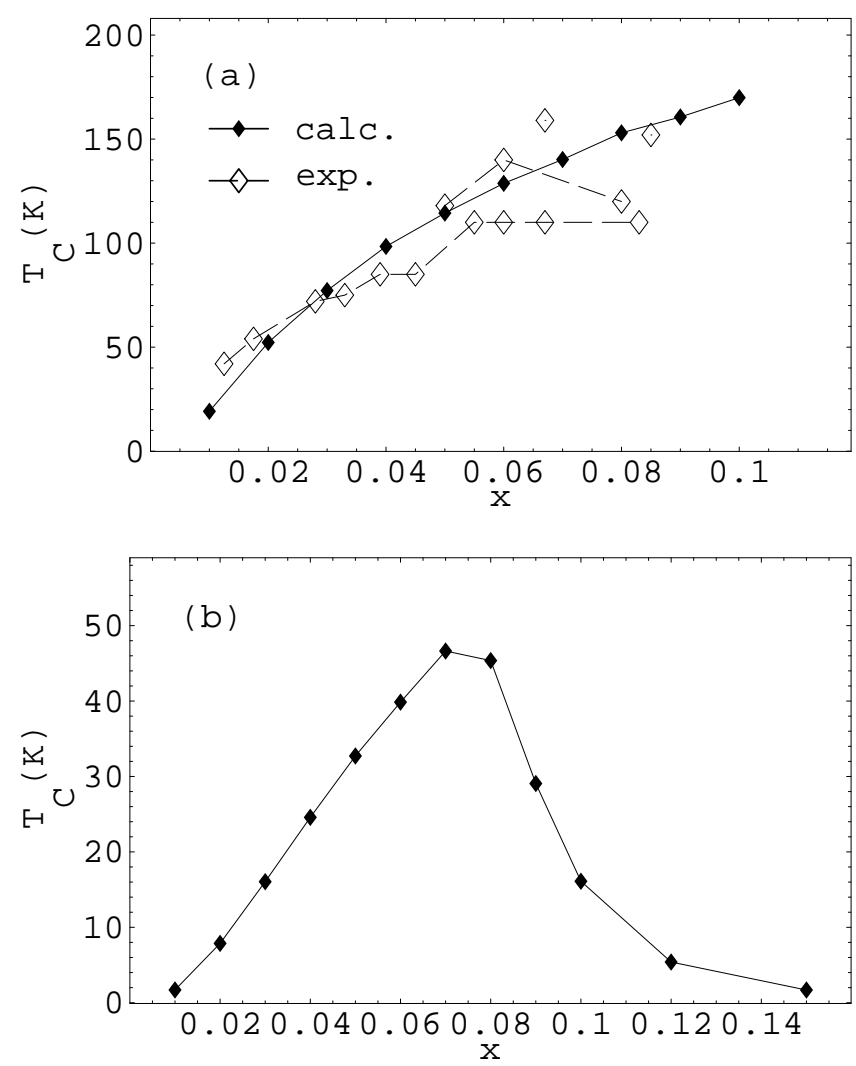

FIG. 3: Calculated Curie temperature $T_{C}$ of (a) $\mathrm{Ga}_{1-x} \mathrm{Mn}_{x}$ As (compared with experimental values of annealed samples ${ }^{5.6 .24 .25}$ ) and (b) $\mathrm{Ga}_{1-x} \mathrm{Mn}_{x} \mathrm{~N}$ for various concentrations $x$ of $\mathrm{Mn}$ ions

should not play an important role for the ferromagnetic stability, which can be easily seen by considering the case of nearest-neighbor interaction only 20 .

\section{SUMMARY}

In this paper, we presented a method for calculating the magnetic properties of ferromagnetic DMS. The method applies a Tyablikov-like approximation for systems with positional disorder to an effective Heisenberg Hamiltonian, whose exchange parameters where obtained by first-principle calculations. Unlike in MFA or VCARPA, no approximations with respect to the positional disorder are made apart from the simplification of a uniform magnetization. As the main advantage over classical MC simulations, the presented treatment of the effective Heisenberg model admits quantum spins and thus may open up a way towards a fully quantum-mechanical treatment of magnetism in DMS. Furthermore, the numerical effort is fairly low compared to MC simulations.

Our calculations of $T_{C}$ for $\mathrm{Ga}_{1-x} \mathrm{Mn}_{x}$ As show excellent agreement with experimental data. For $\mathrm{Ga}_{1-x} \mathrm{Mn}_{x} \mathrm{~N}$, we obtained very low Curie Temperatures despite high effective nearest-neighbor exchange parameters, which 


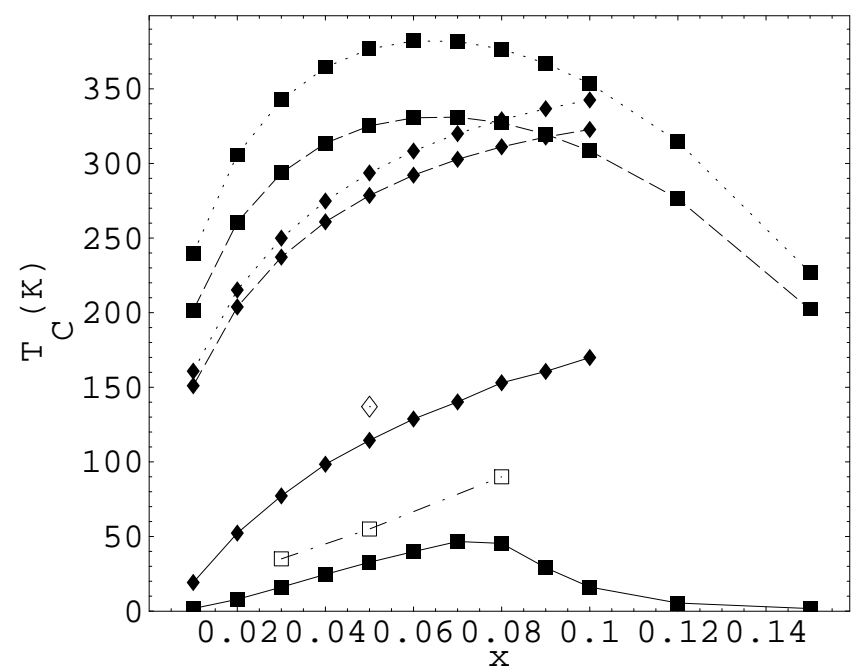

FIG. 4: Comparison of the Curie temperatures $T_{C}$ of $\mathrm{Ga}_{1-x} \mathrm{Mn}_{x} \mathrm{As}$ (diamonds) and $\mathrm{Ga}_{1-x} \mathrm{Mn}_{x} \mathrm{~N}$ (squares) obtained by the presented approach (solid line, filled symbols), VCA-RPA (dashed line, filled symbols), MFA (dotted line, filled symbols) and MC (dash-dotted line, open symbols, taken from $\left.{ }^{15}\right)$.

shows the importance of percolation effects. Moreover, for both $\mathrm{Ga}_{1-x} \mathrm{Mn}_{x}$ As and $\mathrm{Ga}_{1-x} \mathrm{Mn}_{x} \mathrm{~N}$, the $T_{C}$ values we found are much lower than MFA and VCA-RPA values. These results support recent findings obtained by using MC simulations in combination with first-principle methods 15.16 .

The presented model should be improved by using a self-consistent method describing the electronic degrees of freedom at finite temperature (such as, e.g., in ${ }^{32.33}$ ). In order to obtain a fully quantum mechanical theory, quantum spins should be used instead of classical spins in in the calculation of the effective exchange parameters from the electronic structure. This will also remove the ambiguity in the choice of $S$. Furthermore, the treatment of the effective Heisenberg model may be extended to allow for a site-dependent $\left\langle S_{i}^{z}\right\rangle$. In addition, the model might be improved in order to handle systems with a ground state deviating from a saturated ferromagnet. Furthermore, clustering and other forms of short-range chemical ordering may also be included into the model in order to investigate their effects on the magnetic stability. Finally, the method should be applied to other DMS.

\section{Acknowledgments}

This work benefited from the support of the SFB290 of the Deutsche Forschungsgemeinschaft. Thanks to J. Kudrnovský for supplying the exchange parameters and for helpful discussion.
* Electronic address: hilbert@mpa-garching.mpg.de

1 H. Ohno, J. Magn. Magn. Mater. 200, 110 (1999).

2 T. Dietl, Semicond. Sci. Technol. 17, 377 (2002).

3 I. Zutic̀, J. Fabian, and S. Das Sarma, Rev. Mod. Phys. 76, 323 (2004).

${ }^{4}$ M. L. Reed, N. A. El-Masry, H. H. Stadelmaier, M. K. Ritums, M. J. Reed, C. A. Parker, J. C. Roberts, and S. M. Bedair, Appl. Phys. Lett. 79, 3473 (2001).

${ }^{5}$ K. W. Edmonds, K. Y. Wang, R. P. Campion, A. C. Neumann, N. R. S. Farley, B. L. Gallagher, and C. T. Foxon, Appl. Phys. Lett. 81, 4991 (2002).

${ }^{6}$ K. C. Ku, S. J. Potashnik, R. F. Wang, S. H. Chun, P. Schiffer, N. Samarth, M. J. Seong, A. Mascarenhas, E. Johnston-Halperin, R. C. Myers, et al., Appl. Phys. Lett. 82, 2302 (2003).

7 Y. D. Park, A. T. Hanbicki, S. C. Erwin, C. S. Hellberg, J. M. Sullivan, J. E. Mattson, T. F. Ambrose, A. Wilson, G. Spanos, and B. T. Jonker, Science 295, 651 (2002).

8 T. Dietl, H. Ohno, F. Matsukura, J. Cibert, and D. Ferrand, Science 287, 1019 (2000).

9 J. Kudrnovský, I. Turek, V. Drchal, F. Máca, P. Weinberger, and P. Bruno, Phys. Rev. B 69, 115208 (2004).

10 G. Bouzerar and T. P. Pareek, Phys. Rev. B 65, 153203 (2002).

11 V. I. Litvinov and V. K. Dugaev, Phys. Rev. Lett. 86, 5593 (2001).

12 A. Kaminski, V. M. Galitski, and S. Das Sarma, Phys. Rev. B 70, 115216 (2004).
13 J. Schliemann, J. König, and A. H. MacDonald, Phys. Rev. B 64, 165201 (2001).

14 L. Brey and G. Gómez-Santos, Phys. Rev. B 68, 115206 (2003).

15 L. Bergqvist, O. Eriksson, J. Kudrnovský, V. Drchal, P. Korzhavyi, and I. Turek, Phys. Rev. Lett. 93, 137202 (2004).

16 K. Sato, W. Schweika, P. H. Dederichs, and H. KatayamaYoshida, Phys. Rev. B 70, 201202(R) (2004).

17 J. Schliemann, Phys. Rev. B 67, 045202 (2003).

18 C. Timm, J. Phys.: Condens. Matter 15, R1865 (2003).

19 S. Hilbert, Diplomarbeit, Humboldt-Universität zu Berlin (2003).

20 S. Hilbert and W. Nolting, Phys, Rev. B 70, 165203 (2004).

21 A. A. Bogolyubov and S. V. Tyablikov, Dokl. Akad. Nauk SSSR 126, 53 (1959).

22 G. Bouzerar, T. Ziman, and J. Kudrnovský, http://www.arxiv.org/abs/cond-mat/0405322 (2004).

23 J. Kudrnovský (2004), (private communications).

24 S. J. Potashnik, K. C. Ku, R. Mahendiran, S. H. Chun, R. F. Wang, N. Samarth, and P. Schiffer, Phys. Rev. B 66, 012408 (2002).

${ }^{25}$ K. W. Edmonds, P. Bogusławski, K. Y. Wang, R. P. Campion, S. N. Novikov, N. R. S. Farley, B. L. Gallagher, C. T. Foxon, M. Sawicki, T. Dietl, et al., Phys. Rev. Lett. 92, 037201 (2004).

${ }^{26}$ N. Theodoropoulou, A. F. Hebard, M. E. Overberg, C. R. Abernathy, S. J. Pearton, S. N. G. Chu, and R. G. Wilson, 
Appl. Phys. Lett. 78, 3475 (2001).

27 M. E. Overberg, C. R. Abernathy, S. J. Pearton, N. A. Theodoropoulou, K. T. McCarthy, and A. F. Hebard, Appl. Phys. Lett. 79, 1312 (2001).

28 S. Sonodaa, S. Shimizua, T. Sasakib, Y. Yamamotob, and H. Hori, Journal of Crystal Growth 237-239, 1358 (2002).

${ }^{29}$ G. T. Thaler, M. E. Overberg, B. Gila, R. Frazier, C. R. Abernathy, S. J. Pearton, J. S. Lee, S. Y. Lee, Y. D. Park, Z. G. Khim, et al., Appl. Phys. Lett. 80, 3964 (2002).

${ }^{30}$ K. H. Ploog, S. Dhar, and A. Trampert, J. Vac. Sci. Technol. B 21, 1756 (2003).

31 D. Stauffer, Perkolationstheorie: eine Einführung (VCH Verlagsgesellschaft mbH, Weinheim, 1995).

32 W. Nolting, S. Rex, and S. Mathi Jaya, J. Phys.: Condens. Matter 9, 1301 (1997).

33 C. Santos and W. Nolting, Phys. Rev. B 65, 144419 (2002). 